\title{
Prevenção de lesão por pressão em UTI - aplicabilidade da Escala de Braden
}

\author{
Prevention of Injury in ICU - applicability of the Braden Scale \\ Prevención de lesión por presión en UTI - aplicabilidad de la Escala de Braden
}

Renata Gonçalves Vargas ${ }^{1 *}$, Leonardo Pereira do Santos ${ }^{2}$

Como citar esse artigo. Vargas, RG; do Santos, LP. Prevenção de lesão por pressão em UTI - aplicabilidade da Escala de Braden . Revista PróUniverSUS. 2019 Jan./Jun.; 10 (1): $162-165$.

\section{Resumo}

Objetiva-se identificar os fatores de risco que levam o desenvolvimento de Lesão por pressão em pacientes hospitalizados no setor de UTI. Trata-se de uma análise reflexiva a partir de artigos pesquisados na Biblioteca Virtual de Saúde (BVS) e as Bases LILACS, MEDLINE, BDENF e Google Acadêmico. Os resultados apontaram que cuidados com a integridade da pele, como a prevenção de lesões, constitui-se um importante elemento da prática do enfermeiro, auxiliando na identificação de possíveis fatores de risco que podem levar o desenvolvimento de lesões por pressão em pacientes da UTI. Em síntese, medidas preventivas, tais como ações de avaliação de risco e Escala de Braden auxiliam os profissionais para prevenção de lesões por pressão.

Palavras-chave: Lesão por Pressão, Prevenção, UTI.

\begin{abstract}
The objective of this study is to identify the risk factors that lead to the development of pressure-related injury in hospitalized patients in the ICU sector. This is a reflexive analysis from articles researched in the Virtual Health Library (VHL) and the Bases LILACS, MEDLINE, BDENF and Google Scholar. The results indicated that care with skin integrity, such as injury prevention, is an important element of nurses' practice, helping to identify possible risk factors that may lead to the development of pressure injuries in ICU patients. In summary, preventive measures such as risk assessment actions and the Braden Scale help professionals to prevent pressure injuries.

Keywords: Pressure Injury, Prevention, ICU.

\section{Resumen}

Se pretende identificar los factores de riesgo que llevan el desarrollo de Lesión por presión en pacientes hospitalizados en el sector de UTI. Se trata de un análisis reflexivo a partir de artículos investigados en la Biblioteca Virtual de Salud (BVS) y las Bases LILACS, MEDLINE, BDENF y Google Académico. Los resultados apuntaron que el cuidado de la integridad de la piel, como la prevención de lesiones, constituye un importante elemento de la práctica del enfermero, ayudando en la identificación de posibles factores de riesgo que pueden llevar al desarrollo de lesiones por presión en pacientes de la UTI. En síntesis, medidas preventivas, tales como acciones de evaluación de riesgo y Escala de Braden ayudan a los profesionales para la prevención de lesiones por presión.
\end{abstract}

Palabras clave: Lesión por Presión, Prevención, UCI.

1 Enfermeira. Especialista em Enfermagem em UTI Universidade de Vassouras. RJ, Email: renatavargasenf@yahoo.com.br ORCID: https://orcid.org/0000-0002-1038-4429

2Enfermeiro. Especilista em UTI Adulto e Alta Complexidade Hospital Universitário de Vassouras. MBA em Gestão Hospitalar pela UNILEA e CCIH pela UNIFOA. Docente do

Curso de Especialização em Enfermagem em UTI da Universidade de Vassouras, RJ, Brasil. Email: leopsantos2012@yahoo.com.br ORCID: https://orcid.org/0000-0002-0394-5814 


\section{Introdução}

Uma das consequências mais comuns resultante da permanência da hospitalização em especial no setor de UTI, é o aparecimento de lesões na pele que acometem pacientes hospitalizados, o que os tornam mais vulneráveis. Tais lesões na pele são chamadas lesões por pressão (LPP) anteriormente definida como úlcera por pressão (UPP), um dano localizado na pele como resultado da pressão intensa e/ou prolongada em combinação com o cisalhamento. Essa incidência tende ainda a aumentar com a combinação de fatores que acentuam o risco, dentre eles idade, estado nutricional deficitário, obesidade e temperatura ${ }^{1}$.

A LPP é uma lesão localizada na pele e / ou tecido subjacente, normalmente sobre uma proeminência óssea, em resultado da pressão ou de uma combinação entre esta e um cisalhamento ${ }^{2}$.

Dados epidemiológicos evidenciam que a taxa de incidência e prevalência dessas lesões é maior nas UTIs e a partir desses dados que a Organização Mundial da Saúde utiliza como indicador para determinar a qualidade dos cuidados prestados ${ }^{3}$.

Os profissionais que cuidam diretamente de pacientes em estado crítico e que se preocupam com a prevenção de LPP podem encontrar na literatura ferramentas ou escalas que auxiliam na identificação de fatores de risco presentes no paciente. A Escala de Braden é a mais empregada mundialmente, na qual utiliza escores que podem variar de 6 a 23 pontos. Assim, quanto mais baixa a pontuação maior o risco do paciente desenvolver LPP. Foi estabelecido por Braden que na UTI, essa avaliação deve ser feita na admissão, novamente em 48 horas, e após a cada dia ${ }^{4}$.

As LPP são consideradas como eventos adversos ocorridos no processo de hospitalização, que refletem de forma indireta a qualidade do cuidado prestado ${ }^{5}$.

Torna-se necessário a instrumentalização do profissional no qual está diretamente ligado ao processo de cuidados, instrumento esse que o capacitará a colocar em prática procedimentos que irão minimizar os fatores de risco que levam o surgimento das úlceras bem como seus agravos quando a instalação for inevitável. O conhecimento das medidas de prevenção e características da úlcera por pressão deve fazer parte do rol de conhecimento dos profissionais da área de enfermagem ${ }^{6}$.

A instrumentalização da equipe de enfermagem que assiste paciente sob risco de desenvolver úlcera por pressão é essencial, assim o uso da escala de Branden, como instrumento para detectar os pacientes em risco de desenvolver úlcera por pressão em UTI, pode auxiliar na implementação de medidas preventivas de LPP tendo em vista a melhora da qualidade da assistência desenvolvida ${ }^{7}$.
Vale ressaltar que a Escala de Braden permite a padronização da avaliação de risco para LPP, auxiliando tanto na identificação dos fatores de risco, como na prescrição das medidas preventivas. Ainda pode auxiliar para redução da variação da avaliação de risco entre os enfermeiros, visando evitar e corrigir diferenças, erros e discordâncias na escolha dos escores. Para isso, é preciso que os enfermeiros tenham uma clara compreensão do significado das descrições das subescalas ${ }^{8}$.

Os agravantes para o acometimento de pacientes por LPP podem ser prevenidos, com isso reduz tanto o risco do desenvolvimento como as complicações destas feridas $^{9,10}$.

Assim, diante o exposto, tem-se por objetivo identificar fatores de risco que levam o desenvolvimento de Lesão por pressão em pacientes hospitalizados no setor de UTI a partir da literatura já existente.

\section{Metodologia}

Trata-se de um estudo reflexivo a partir de uma pesquisa bibliográfica desenvolvida com auxílio de material já elaborado, constituído principalmente de livros e artigos científicos .

Os dados foram coletados em Base de Dados Virtuais. Para tal utilizou-se a Biblioteca Virtual de Saúde (BVS), na seguinte Base de informação: Literatura Latino-Americana e do Caribe em Ciências da Saúde (LILACS), Literatura Internacional em Ciência da Saúde (MEDLINE), Base de Dados de Enfermagem (BDENF) e pelo endereço eletrônico scholar.google. com.br, no período de Maio à Julho de 2018.

\section{Resultados e Discussão}

De posse da bibliografia potencial, foi feita a leitura na íntegra dos artigos e posteriormente análise a luz da temática em questão.

\section{Fundamentos para reflexão acerca daLesão por pressão na UTIe escala de Braden}

Estudos que avaliam ações dos profissionais de enfermagem, antes e após utilização de protocolo de prevenção de LPP em UTI. Referem que após uso do protocolo, observou-se maior frequência das ações preventivas, demonstrando a relevância do uso dessa ferramenta na adoção das recomendações baseadas em evidências científicas pelos profissionais ${ }^{1}$.

No estudo em que avaliou a efetividade de uma campanha para prevenção de LPP em um Hospital de Ensino. Os resultados apontaram aumento no percentual de adesão, estatisticamente significativo para reposicionamento, angulação de lateralização e elevação 
de cabeceira e elevação de calcâneos. Destacando melhores índices de adesão para o setor com a equipe mais envolvida nas discussões durante as atividades de capacitação. Havendo efetividade da campanha para os itens melhor discutidos na capacitação. A efetividade deste tipo de abordagem é dependente da participação ativa da equipe na discussão de medidas a serem aplicadas e de recursos disponíveis. Faz-se necessária uma abordagem contínua e sistemática ${ }^{2}$.

A literatura ainda aponta que ao determinar que fatores influenciaram no aparecimento $\mathrm{e}$ desenvolvimento, verificando incidência e prevalência em 155 pacientes. Onde dezoito pacientes apresentavam LPP na admissão e 40 a desenvolveram durante a internação, totalizando 125 LPP. A prevalência foi de $37,41 \%$ e a incidência de $25,8 \%$. O aparecimento de novas lesões ocorreu, em média, no $7^{\circ}$ dia de internação. Dos pacientes internados $79 \%$ mantiveram-se estáveis e/ou melhorados. Na população estudada as lesões apresentaram prevalência de $37,41 \%$ e incidência de $25,8 \%$. A aplicação do protocolo de LPP foi eficaz em $79 \%$ dos pacientes, sendo que apareceram com maior frequência nos pacientes mais graves ${ }^{3}$.

Buscando avaliar a validade preditiva dos escores da escala de Braden em pacientes de um Centro de Terapia Intensiva e descrever as medidas preventivas implementadas pela equipe de enfermagem. Como resultado dos testes de validade preditiva apontou os escores 14,13 e 12 como os mais eficientes na predição de risco para úlcera por pressão, nas primeira, segunda e terceira avaliações com seus respectivos valores de sensibilidade $(95 \%, 95 \%$ e $94 \%)$ e especificidade $(45 \%$, $55 \%$ e $77 \%$ ). Apontando a escala de Braden como um instrumento eficiente para identificar precocemente o risco e para subsidiar a elaboração de um plano de cuidado capaz de prevenir danos na pele de pacientes em estado críticos ${ }^{4}$.

No estudo que buscou investigar risco de acometimento de LPP no cliente, e a validade do cuidado de enfermagem aplicando a escala de Braden para verificar as evidências desse cuidado na sua incidência. Encontrou-se 11 clientes com LPP, numa incidência de $26,83 \%$. A maioria $(57,1 \%)$ de úlcera encontra-se no estágio I. Clientes com LPP têm escores menores que os sem LPP mostrando sensibilidade para predizer o risco de LPP. A evidência dos cuidados de enfermagem foi demonstrada pela densidade de 47,12 $\%$ para os clientes sem LPP; a incidência das LPP foi menor que as encontradas em outras UTIs; os cuidados de enfermagem preventivos reduzem as LPP ${ }^{5}$.

Ao Avaliar o efeito de intervenções educativas, no nível de conhecimento dos membros da equipe de enfermagem sobre a prevenção de úlceras por pressão em um Centro de Terapia Intensiva. Sete enfermeiros participaram somente da fase pré-intervençãoe obtiveram $86,4 \%$ de acertos no total de questões. Os auxiliares e técnicos de enfermagem na fase pré-intervenção $(\mathrm{n}=25)$ obtiveram $74,3 \%$ de acertos e na fase pósintervenção $(n=36)$ apresentaram melhora com $81,2 \%$ de acertos. Contudo as estratégias utilizadas não foram totalmente eficazes, onde em algumas questões os acertos pósintervenção foram abaixo de $70 \%$. Demandando novas intervenções que precisam ser realizadas tendo em vista uma maior adesão dos profissionais ao programa educativo e melhorar o conhecimento sobre o tema ${ }^{6}$.

Ao procurar identificar os pacientes com risco de desenvolverúlcera por pressão LPP em unidade de terapia intensiva UTI por meio da Escala de Braden e relacionar esses escores com a assistência de enfermagem na sua prevenção. Um estudo apontou que houve prevalência de pacientes com risco moderado e idade média de 54,35 anos. A relação entre a classificação de risco e as medidas preventivas não estavam ondizentes com os escores encontrados, sendo a mudança de decúbito e o uso de coxins, os cuidados menos observados. A Escala de Braden foi importante, uma excelente ferramenta para subsidiar a assistência de enfermagem na prevenção de LPP em UTI ${ }^{7}$.

E ao descrever o processo de instrumentalização para auxiliares técnicos de enfermagem e familiares na prevenção de LPP em pacientes de uma UTI adulto. Após a orientação para avaliação dos pacientes em risco, os cuidados são 'simples e fáceis' e a escala de Braden, utilizada para avaliar o paciente em risco, é de 'fácil aplicação'. Para a maioria dos familiares e/ou cuidadores, pode-se perceber a satisfação em poder, com medidas simples, evitar a úlcera por pressão e fornecer conforto ao seu familiar ${ }^{8}$.

\section{Conclusão}

$\mathrm{O}$ estudo buscou identificar fatores de risco que levam o desenvolvimento de Lesão por pressão em pacientes hospitalizados no setor de UTI.

Verificou-se a relevância de se utilizar a classificação da Escala de Braden nos cuidados prestados na UTI, devendo ser compartilhada nos diferentes turnos de trabalho entre os enfermeiros para a prevenção da LPP.

De acordo com a literatura consultada, cuidados com a integridade da pele, como a prevenção de lesões, constitui-se um importante elemento da prática do enfermeiro, auxiliando na identificação de possíveis fatores de risco que podem levar o desenvolvimento de LPP em pacientes da UTI.

Medidas preventivas, tais como ações de avaliação de risco e Escala de Braden auxilia os profissionais para prevenção de LPP.

Por fim, a educação continuada foi apontada como um forte aliado na prevenção de LPP. Assim, aperfeiçoar-se, torna-se passo fundamental para que 
os cuidados prestados pela enfermagem no âmbito da Unidade de Terapia Intensiva, seja pautado na assistência de qualidade ao paciente hospitalizado.

\section{Referências}

1. Vasconcelos JMB. Ações de enfermagem antes e após um protocolo de prevenção de lesões por pressão em terapia intensiva. Esc. Anna Nery. 2017; 21(1):1-9. Disponível: http://www.scielo.br/scielo.php?pid=S141414 52017000100201\&script=sci_abstract\&tlng=es

2. Olkoski E. Aplicação de Medidas de Prevenção para úlceras por pressão pela equipe de enfermagem antes e após uma campanha educativa. Esc. Anna Nery. 2016; Abr./Jun. 20 (2): 363-369. Disponível: http:// www.scielo.br/scielo.php?pid=S1414-81452017000100201\&script $=$ sci abstract\&tlng=es

3. Louro M. Avaliação de Protocolo de Prevenção e Tratamento de Úlceras de Pressão. Revista Brasileira de Terapia Intensiva 2007; Jul./Set; 19 (3): 337-341.

4. Costa IG, Caliri MHL. Validade preditiva da escala de Braden para pacientes de terapia intensiva. Acta Paul Enferm 2011; 24 (6): 772-777. Disponível em: http://www.redalyc.org/html/3070/307023880007/

5. Sousa CA, Santos I, Silva LD. Aplicando recomendações da Escala de Braden e prevenindo úlceras por pressão - evidências do cuidar em enfermagem. Rev Bras Enferm 2006 maio-jun; 59(3): 279-84. Disponível em: http://www.redalyc.org/html/2670/267019621005/

6. Fernandes LM, Caliri MHL, Haas VJ. Efeito de intervenções educativas no conhecimento dos profissionais de enfemagem sobre prevenção de úlceras pressão. Acta Paul Enferm 2008;21(2):305-11. Disponível em: http://www.redalyc.org/html/3070/307023824012/

7. Lise Fernanda, Silva LC. Prevenção de úlcera por pressão: instrumentalizando a enfermagem e orientando o familiar cuidador. Acta Sci. Health Sci. Maringá, v. 29, 2007;29(2):85-89. Disponível em: http://www. redalyc.org/html/3072/307226621001/

8. Barbosa TP, Beccaria LM, Poletti NAA. Avaliação do risco de úlcera por pressão em UTI e assistência preventiva de enfermagem. Rev enferm UERJ, Rio de Janeiro, 2014 mai/jun; 22(3):353-8. Disponível em: http:// www.e-publicacoes.uerj.br/index.php/enfermagemuerj/article/view/13724

9. Moreira PSCR, Souza MMT. Cuidados de enfermagem a lesão por pressão - relato de caso. Revista Pró-UniverSUS. 2018 Jan./Jun.; 09 (1): 105110. Disponível em: http://editora.universidadedevassouras.edu.br/index. $\mathrm{php} / \mathrm{RPU} /$ article/view/1270

10. Ferreira TMC, de Lima CLJ, Ferreira JDL, de Oliveira PS, Agra G, Ferreira IMC, Costa MML. Conhecimento de enfermeiros sobre o uso da colagenase em lesões por pressão. Revista de Enfermagem UFPE on line 2018; 12(1):128-136. Disponível em: https://periodicos.ufpe.br/revistas/ revistaenfermagem/article/view/23190 